\title{
Manipulative therapy and a low load exercise regimen each reduced the frequency and intensity of cervicogenic headache
}

Jull $G$, Trott P,Potter $H$, et al. A randomized controlled trial of exercise and manipulative therapy for cervicogenic headache. Spine 2002;27:1835-43.

\section{QUESTION: In patients with cervicogenic headache, does manipulative therapy and/or specific low load exercise reduce headaches?}

\section{Design}

Randomised (allocation concealed*), blinded (outcome assessors),* controlled trial with follow up immediately after treatment and at 12 months.

\section{Setting}

5 centres in Australia.

\section{Patients}

200 patients (mean age 37 y, 70\% women) with cervicogenic headache, defined as unilateral (or unilateral dominant-side consistent) headache associated with neck pain and aggravated by neck postures or movement, joint tenderness in $\geq 1$ of the upper 3 cervical joints, and headache frequency of $\geq 1$ per week over a period of 2 months to 10 years. Exclusion criteria were bilateral headaches, features suggestive of migraine, contraindications to manipulative therapy, involvement in litigation or workers' compensation, and physiotherapy or chiropractic treatment for headache in the previous 12 months. Follow up at 12 months was $97 \%$.

\section{Intervention}

51 patients were allocated to manipulative therapy (MT), which included low velocity cervical joint mobilisation and high velocity manipulation. 52 patients were allocated to therapeutic exercise (ExT), which used low load endurance exercises to train muscle control of the cervicoscapular region. 49 patients were allocated to a combination of MT and ExT (combined therapy), and 48 were allocated to no physical therapy interventions (control). Active treatment involved a minimum of 8 and a maximum of 12 treatments ( $\leq 30 \mathrm{~min} / \mathrm{session})$ delivered by experienced physiotherapists over a 6 week period.

\section{Main outcome measures}

Main outcome was change in headache frequency from baseline to immediately after treatment and at 12 months. Secondary outcomes included changes in headache intensity and duration.

\section{Main results}

Analysis was by intention to treat. MT, ExT, and combined therapy reduced headache frequency more than the control therapy immediately after the intervention (7 wks) and at 12 months (table). Similar results were found for headache intensity. The 3 active treatments did not differ from each other for headache frequency or intensity.

MT and ExT did not differ from the control group for headache duration at 12 months. Combined therapy reduced headache duration more than the control condition at 7 weeks $(4.25 v 2.13 \mathrm{~h}$ in past wk, $\mathrm{p}<0.001)$ and 12 months $(4.26 v 2.01 \mathrm{~h}, \mathrm{p}<0.05)$. Combined therapy reduced headache duration more than ExT at 7 weeks and 12 months.

\section{Conclusions}

In patients with cervicogenic headache, manipulative therapy and a low load exercise regimen each reduced headache frequency and intensity more than no physical therapy. A combination of manipulative therapy and exercise was not better than each individual therapy for these outcomes.

*See glossary.

website extra Additional information appears on the Evidence-Based Medicine website www.evidence-based medicine.com

Sources of funding: National Health and Medical Research Council; Physiotherapy Research Foundation; University of Queensland Foundation; St. Vincent's Foundation; Centre of National Research on

Disability and

Rehabilitation Medicine.

For correspondence: Dr G Jull, University of Oueensland, Brisbane, Oueensland, Australia g.jull@shrs.uq.edu.au

Manipulative therapy (MT), therapeutic exercise (ExT), and MT + ExT (combined) v control for cervicogenic headachet

\begin{tabular}{llll}
$\begin{array}{l}\text { Outcomes at } 12 \\
\text { months }\end{array}$ & Comparison & Mean change & $\begin{array}{l}\text { Mean difference } \\
\text { between groups } \\
(95 \% \mathrm{Cl})\end{array}$ \\
$\begin{array}{c}\text { Headache frequency } \\
(\mathrm{d} \text { in past wk) }\end{array}$ & MT $v$ control & $2.25 v 0.95$ & $1.3(0.58$ to 2.02$)$ \\
\hline & ExT $v$ control & $2.52 v 0.95$ & $1.57(0.91$ to 2.23$)$ \\
\hline & Combined $v$ control & $2.12 v 0.95$ & $1.17(0.52$ to 1.82$)$
\end{tabular}

$\mathrm{tCl}$ defined in glossary; mean difference between groups and $\mathrm{Cl}$ calculated from data in article.

\section{COMMENTARY}

The study by Jull $e$ al is the most rigorous attempt to date to assess the effects of physical therapies on the common clinical problem of cervicogenic headache. Its multicentre design, as well as some flexibility in the number and content of treatment sessions, increase the generalisability of the results to clinical practice. 12 month follow up adequately tested the durability of responses. Blinding was possible only for outcome assessment, but the success of this blinding was not reported.

The results indicate a superior effect of manipulative and exercise therapies used alone and in combination compared with a control condition. On balance, it seems that combined therapy offers slightly more than either therapy alone. The results are consistent with a review, which showed that multimodal manual therapy, including exercise, is superior to certain physical medicine modalities, rest, and control treatments for cervicogenic headache.

It is impossible to determine the contribution of the non-specific effect of repeated contact with therapists. A course of 8-12 treatment sessions over a 6 week period was given to active treatment groups, but not to the control group. None the less, active treatments worked, and 2 active treatments worked a little better than one. No explanation for the limits on the number of treatment sessions was provided. Only $12-21 \%$ of patients in the active treatment groups sought additional treatment in the follow up period, suggesting that $\leq 12$ treatments is sufficient. However, is $<8$ treatments effective? A small trial of manipulation for cervicogenic headache showed significant improvements from baseline with 6 treatments, but these were not better than the active comparator of laser and deep friction massage; there was no non-intervention group. ${ }^{2}$

Practising clinicians should take note of the trial's selection criteria of unilateral or predominantly unilateral headache with neck pain and upper cervical tenderness to guide their selection of patients who may benefit from these treatments. Should there be angst about the potential (small) risk of complications of cervical manipulation, exercise therapy alone would still be effective, or the manual therapy component could be limited to low velocity mobilisation.

Michael Yelland, MBBS, FRACGP University of Queensland Herston, Queensland, Australia

1 Gross A, Kay T, Hondras M, et al. Manual therapy for mechanical neck disorders: a systematic review Man Ther $2009 ; 7: 131$. 149 .

Nilsson N. A randomized controlled trial of the effect of spinal manipulation in the treatment of cervicogenic headache. J Manipulative Physiol Ther 1995;18:435-40. 